Revue internationale P.M.E.

Économie et gestion de la petite et moyenne entreprise

\title{
Les petites entreprises et les petits entrepreneurs en Espagne : une approche qualitative
}

\section{Joaquin Guzmân Cuevas}

Volume 8, numéro 1, 1995

URI : https://id.erudit.org/iderudit/1008278ar

DOI : https://doi.org/10.7202/1008278ar

Aller au sommaire du numéro

\section{Éditeur(s)}

Presses de l'Université du Québec

ISSN

0776-5436 (imprimé)

1918-9699 (numérique)

Découvrir la revue

\section{Citer cette note}

Guzmân Cuevas, J. (1995). Les petites entreprises et les petits entrepreneurs en Espagne : une approche qualitative. Revue internationale P.M.E., 8(1), 121-137. https://doi.org/10.7202/1008278ar

\section{Résumé de l'article}

Dans cet article, l'auteur effectue une analyse de l'entrepreneur espagnol en distinguant la structure entrepreneuriale et les caractéristiques propres à l'entrepreneur. L'étude de ces deux aspects de l'appareil productif espagnol devrait nous permettre d'expliquer les causes qui sont à l'origine de ses principales faiblesses : un faible niveau d'exportation et de transfert technologique, une excessive dépendance au crédit bancaire, ainsi que des coûts salariaux élevés.

D'un point de vue de la structure entrepreneuriale, il existe trois raisons pouvant expliquer la situation actuelle : a) une spécialisation dans les secteurs industriels de faible demande, b) une forte atomisation dans le secteur des services, et conjointement, un nombre élevé d'entreprises participant faiblement à la création de PIB et d'emplois, c) une prédominance de petites entreprises « indépendantes ", peu sensibles à la conjoncture économique générale, si bien qu'elles réussissent à maintenir leur activité, malgré leur faible niveau de compétitivité. À ces causes s'ajoutent les faiblesses caractéristiques des petits entrepreneurs de la majeure partie d'Espagne. Paradoxalement, les régions les moins avancées ne manquent pas de dirigeants. De ce fait, le problème semble se poser en termes qualitatifs et non quantitatifs. En effet, la situation actuelle ne remet pas en cause la formation entrepreneuriale, mais plutôt l'élément attenant aux caractéristiques internes de " l'entrepreneur », c'est-à-dire la sphère « impulsive » de la fonction entrepreneuriale. Car, à l'heure actuelle, la majeure partie des micro-entreprises et des petites entreprises se créent afin de répondre à des exigences de survivance économique.

D’un point de vue méthodologique, ce travail tente de souligner la nécessité de relier Tanalyse de l'entreprise á celle de I'entrepreneur, afin de développer des politiques d'appui aux PME plus efficaces que celles qui existent actuellement.
Ce document est protégé par la loi sur le droit d'auteur. L'utilisation des services d’Érudit (y compris la reproduction) est assujettie à sa politique d'utilisation que vous pouvez consulter en ligne.

https://apropos.erudit.org/fr/usagers/politique-dutilisation/ 


\title{
Les petites entreprises et les petits entrepreneurs en Espagne: une approche qualitative
}

\author{
Joaquín GUZMÁN CUEVAS* \\ Université de Séville
}

MOTS CLÉS
Entrepreneur - Micro-entreprises - Tissu entrepreneurial Fonction entrepreneuriale - Politiques d'appui - PME

\begin{abstract}
RÉSUMÉ
Dans cet article, l'auteur effectue une analyse de l'entrepreneur espagnol en distinguant la structure entrepreneuriale et les caractéristiques propres à l'entrepreneur. L'étude de ces deux aspects de l'appareil productif espagnol devrait nous permettre d'expliquer les causes qui sont à l'origine de ses principales faiblesses: un faible niveau d'exportation et de transfert technologique, une excessive dépendance au crédit bancaire, ainsi que des coûts salariaux élevés.

D'un point de vue de la structure entrepreneuriale, il existe trois raisons pouvant expliquer la situation actuelle : a) une spécialisation dans les secteurs industriels de faible demande, b) une forte atomisation dans le secteur des services, et conjointement, un nombre élevé d'entreprises participant faiblement à la création de PIB et d'emplois, c) une prédominance de petites entreprises "indépendantes", peu sensibles à la conjoncture économique générale, si bien qu'elles réussissent à maintenir leur activité, malgré leur faible niveau de compétitivité. À ces causes s'ajoutent les faiblesses caractéristiques des petits entrepreneurs de la majeure partie d'Espagne. Paradoxalement, les régions les moins avancées ne manquent pas de dirigeants. De ce fait, le problème semble se poser en termes qualitatifs et non quantitatifs. En effet, la situation actuelle ne remet pas en cause la formation entrepreneuriale, mais plutôt l'élément attenant aux caractéristiques internes de "l'entrepreneur", c'est-à-dire la sphère "impulsive" de la fonction entrepreneuriale. Car, à l'heure actuelle, la majeure partie des micro-entreprises et des petites entreprises se créent afin de répondre à des exigences de survivance économique.
\end{abstract}

* Joaquín Guzmán Cuevas est professeur titulaire d'économie appliquée et directeur du Groupe de recherche - la petite et moyenne entreprise - Université de Séville, Espagne. Adresse : Département d'économie appliquée, Université de Séville, Ramón y Cajal, 1, 41018 Séville, Espagne. 
D'un point de vue méthodologique, ce travail tente de souligner la nécessité de relier l'analyse de l'entreprise à celle de l'entrepreneur, afin de développer des politiques d'appui aux PME plus efficaces que celles qui existent actuellement.

\section{ABSTRACT}

In this article, the author analyzes separately the Spanish entrepreneurial structure and the characteristics of the entrepreneur in order to delve into the causes of the weaknesses of the productive system : low level of exportations and technology, excessive dependence on credit banking and high labour costs.

From the entrepreneurial structural point of view, three reasons could explain this situation : a) industrial specialization in activities of weak demand, b) great "atomization" in the service sector, with a lot of businesses whose contribution to GDP and national employment is low, c) prevalence of "independent" small businesses isolated from the economic environment which, therefore, are able to survive in spite of a low level of competitiveness. These reasons are linked to the weaknesses of small entrepreneurs from most Spanish regions. In depressed regions, paradoxically, there is no lack of entrepreneurs. The problem is, basically, qualitative, and its fundamental factor is not to be found in the management sphere but in the internal characteristics of the entrepreneur - called the "booster" sphere of the entrepreneurship. This provokes the creation of micro and small enterprises only for economic survival.

From a methodological point of view, the article tries to show that it is necessary to relate the analysis of the enterprises and that of the entrepreneur in order to improve the SME's support policy.

\section{RESUMEN}

En este artículo, el autor realiza una aproximación analítica al empresariado español a partir de la distinción entre la estructura empresarial y las características del hombre-empresario. Del análisis de ambas vertientes, se intenta profundizar en las causas que originan los puntos débiles del aparato productivo español : bajos niveles de exportación y transferencia tecnológica, excesiva dependencia del crédito bancario y elevados costes laborales.

Desde el punto de vista de la estructura empresarial, tres razones se pueden apuntar para explicar esa situación : a) especialización en sectores industriales de demanda débil, b) gran atomización en el sector servicios, con alto número de empresas pero con poca aportación al PIB y al empleo, c) predominio de pequeñas empresas “independientes " muy aisladas del clima económico y, por consiguiente, capaces de mantener la supervivencia a pesar de no alcanzar altos niveles de competitividad. Estas causas enlazan con las debilidades que presentan los pequeños empresarios en la mayor parte de las regiones españolas. En las menos avanzadas, no se produce, paradójicamente, un problema de escasez de empresarios. El problema es más bien cualitativo, cuyo elemento fundamental no está en la formación sino en las características internas del empresario - dentro de lo que se ha denominado la esfera "impulsora " de la función empresarial - que hacen 
que la mayor parte de las microempresas y pequeñas empresas se creen por razones de supervivencia económica.

Desde el punto de vista metodológico, el trabajo trata de poner de manifiesto la necesidad de analizar y relacionar la problemática de la empresa y las características del hombre-empresario, para alcanzar una política de apoyo a las PyMEs más eficaz que la actual.

\section{Introduction}

L'entrepreneur est l'élément qui donne naissance à l'organisation industrielle et qui la maintient en vie. Pour faire l'analyse de ses caractéristiques fondamentales, nous distinguerons la notion d'entreprise de celle de l'entrepreneur. Le titre de cet article suggère d'ailleurs une distinction méthodologique fondamentale, qui servira à mettre en relief la nature et les causes de la faiblesse générale des PME espagnoles.

Les problèmes de compétitivité, c'est-à-dire la plupart de ceux qui affectent les PME, particulièrement en Espagne, mais aussi ailleurs, sont associés à l'environnement extérieur de l'entreprise : ces problèmes peuvent être de nature financière, fiscale, bureaucratique, informatique, etc. Cependant, en plus des problèmes créés par le milieu externe à l'entreprise, d'autres types de difficultés, ayant pour source la structure organisationnelle représentée par le dirigeant, peuvent se présenter. Le thème de l'entrepreneur, en marge de sa facette de gestion, a très peu fait l'objet d'analyse et ne constitue pas un élément d'étude important de l'histoire de la pensée économique. Même s'il commence à être considéré par les théoriciens, il reste encore hors de la sphère d'étude de l'analyse économique ; certains pourraient objecter que cette notion relève plus de la psychologie et des sciences sociales, cela n'expliquerait tout de même pas ce désintéressement de la part des économistes. Pourtant, il est particulièrement important de considérer l'entrepreneur comme la « matière première » de l'entreprise, surtout lorsqu' on sait que le tissu industriel est composé en majorité de PME, et que dans ce type de structure, la figure de l'entrepreneur constitue le facteur déterminant de l'évolution de l'unité productive.

Aussi, tout en tenant compte de la distinction entrepreneur-entreprise, nous voulons ici analyser brièvement le tissu productif espagnol, car cette étude préalable semble indispensable à l'évaluation des caractéristiques propres aux PME et des problèmes qu'elles doivent résoudre. Dans une troisième partie, nous tenterons de décrire, d'un point de vue qualitatif, les traits caractéristiques du petit entrepreneur. Enfin, nous établirons un bilan critique des politiques de soutien instaurées ces dernières années en Espagne. 
L'objectif de ce travail n'est pas d'effectuer une analyse profonde de chacun des thèmes annoncés. En effet, il s'agit plutôt d'une approximation analytique de chacun d'eux afin de mettre en valeur la nécessité d'un mode d'étude reliant la sphère de l'entreprise à l'entrepreneur et aux politiques appliquées aux PME. Autrement, il nous serait impossible de développer les connaissances empiriques actuelles concernant l'appareil productif et ses possibilités de transformations futures.

\section{Le tissu entrepreneurial espagnol}

D'un point de vue dimensionnel, la structure entrepreneuriale espagnole présente des différences par rapport à son environnement communautaire européen.

La référence la plus adaptée, ou la «moins mauvaise », pour déterminer la taille d'une entreprise est le nombre d'employés. Si l'on considère ce critère d'évaluation, l'Espagne se situe au second rang des pays de la Communauté européenne pour ses micro-entreprises, ce qui signifie, selon le tableau 1 , que $94,86 \%$ des entreprises espagnoles emploient moins de dix salariés. En outre, l'importance relative de ce type de structure entrepreneuriale, à l'exception de l'Irlande $(37,23 \%)$ et du Danemark $(71,86 \%)$, est un des traits caractéristiques des économies européennes.

TABleau 1

Le tissu entrepreneurial dans les pays de la CE (*)

\begin{tabular}{lccccc}
\hline Pays & Groupe 1 & Groupe 2 & Groupe 3 & Groupe 4 & Total \\
\hline Allemagne & 86,04 & 12,87 & 0,92 & 0,17 & 100 \\
Belgique & 94,35 & 5,08 & 0,46 & 0,11 & 100 \\
Danemark & 71,86 & 26,02 & 1,85 & 0,27 & 100 \\
Espagne & 94,86 & 4,81 & 0,29 & 0,04 & 100 \\
France & 93,52 & 5,90 & 0,48 & 0,10 & 100 \\
Grèce & 92,79 & 6,63 & 0,38 & 0,19 & 100 \\
Hollande & 91,71 & 7,33 & 0,65 & 0,12 & 100 \\
Irlande & 37,23 & 53,75 & 8,18 & 0,84 & 100 \\
Italie & 90,60 & 9,12 & 0,24 & 0,04 & 100 \\
Luxembourg & 88,20 & 10,74 & 0,95 & 0,12 & 100 \\
Portugal & 95,19 & 4,41 & 0,35 & 0,05 & 100 \\
Royaume-Uni & 90,09 & 8,75 & 0,99 & 0,18 & 100 \\
\hline
\end{tabular}

* Groupe 1: moins de 10 salariés

Groupe 2: 10-49 salariés

Groupe 3: $50-499$ salariés

Groupe 4: plus de 500 salariés

Source : Communauté européenne, 1991. 
En faisant abstraction du poids quantitatif des micro-entreprises, et en se centrant sur les segments dimensionnels, l'Espagne occupe la troisième place des pays possédant un faible pourcentage d'entreprises de grande taille, après le Portugal et l'Italie. Cela explique l'absence de sociétés espagnoles des classements établissant un palmarès des cent premières entreprises européennes. Sur ce plan, il semble clair que l'atomisation entrepreneuriale espagnole se caractérise plus par la faiblesse du nombre d'unités productives appartenant aux segments de taille supérieure que par le nombre élevé de micro-entreprises. En effet, l'analyse du tableau 1 révèle que le tissu productif espagnol n'est constitué que par $4,81 \%$ de petites entreprises (celles du groupe 2, employant de 10 à 49 salariés), ce qui lui donne l'avant-dernier rang des pays européens. En ce qui concerne les entreprises dites « moyennes », la proportion n'est que de $0,29 \%$. En termes comparatifs, la structure productive espagnole semble être caractérisée par les deux traits suivants : une quasi-absence d'entreprises leaders sur le marché européen et une insuffisance de PME employant plus de 10 salariés. De plus, une grande partie des micro-entreprises sont familiales et présentent un degré de technicité peu élevé. En conséquence, cette excessive atomisation constitue une des causes du retard compétitif de l'appareil productif espagnol, par rapport à ses concurrents européens.

TABleau 2

Distribution des 2000 premières entreprises en Espagne (1990)

\begin{tabular}{lcccc}
\hline Province & $\begin{array}{c}\text { Nombre } \\
\text { d'entreprises }\end{array}$ & $\mathbf{( \% )}$ & $\begin{array}{c}\text { Pourcentage } \\
\text { des ventes }\end{array}$ & $\begin{array}{c}\text { Pourcentage } \\
\text { des emplois }\end{array}$ \\
\hline Madrid & 679 & 33,9 & 53,1 & 54,8 \\
Barcelone & 504 & 25,2 & 20,0 & 18,6 \\
Pays basque & 160 & 8,0 & 5,5 & 5,8 \\
Sous-total & 1343 & 67,1 & 78,6 & 79,2 \\
Reste de l'Espagne & 657 & 32,9 & 21,4 & 20,8 \\
\hline
\end{tabular}

Source : Revista Actualidad Económica.

À cette forte atomisation s'ajoute l'importante concentration des grandes entreprises dans les trois grands pôles industriels espagnols: Madrid, Barcelone et Pays basque. Comme l'indique le tableau 2, c'est dans ces trois centres que sont installés plus des deux tiers des grandes entreprises existant en Espagne et qu'on relève près de $80 \%$ des ventes et des emplois générés par l'économie espagnole. Dans ce cas précis, nous nous référons aux entreprises en tant que telles et non à de simples établissements ou aux centres commerciaux dépendants de celles-ci. Par conséquent, l'atomisation industrielle, que ce soit en termes quantitatifs (nombre d'entreprises) ou qualitatifs (poids de 
celles-ci dans l'économie espagnole), est particulièrement élevée dans le reste de l'Espagne, et plus encore dans les zones économiquement peu avancées. Mais en marge de cette forte atomisation de la population entrepreneuriale et de l'importante concentration géographique des grandes entreprises, les diverses études empiriques réalisées sur le fonctionnement des PME ont souligné leurs principaux points faibles.

- Un faible poids dans le volume des exportations nationales. Depuis la fin des années 80 s'accentue la présence des PME dans les exportations espagnoles, atteignant un pourcentage voisin de $40 \%$ du volume total. Cependant, bien que statistiquement elles apparaissent comme des PME, une bonne part de ce pourcentage provient d'entreprises appartenant au secteur public (Instituto Nacional de Estadística et moyennes entreprises avec participation des administrations régionales ou locales) ou à de grands groupes nationaux ou multinationaux; ce qui ôte une certaine importance à la compétitivité des authentiques PME de caractère autonome dans le commerce extérieur.

- En marge des entreprises de taille moyenne, filiales, ou contrôlées par le secteur public, le transfert technologique se pratique toujours à un haut niveau, celui des grandes entreprises. Actuellement, à l'exception de certains centres industriels, les PME ne possèdent pas les capacités suffisantes pour intégrer de nouvelles technologies dans leur processus de production (Jordá, 1992).

- Les coûts salariaux des micro-entreprises sont particulièrement élevés, étant donné qu'elles constituent la majorité des entreprises du tissu productif espagnol. En effet, selon l'Institut de la petite et moyenne entreprise, les coûts salariaux des entreprises employant moins de 20 salariés représentent $30 \%$ du produit généré par l'activité de la société, alors que pour les grandes entreprises (de plus de 500 employés), ils se situent à $26,2 \%$.

- Les PME sont très dépendantes des crédits bancaires qui leur sont accordés. En effet, les entreprises employant moins de 100 salariés recourent au financement bancaire pour assurer $80 \%$ de leurs ressources propres. En ce qui concerne les grandes entreprises (de plus de 500 salariés), ce pourcentage s'élève à $60 \%$ (Banque d'Espagne, 1990). De plus, cette dépendance financière est accrue par la nature des prêts, qui le plus souvent sont à court terme, ce qui veut dire pour l'entreprise des coûts financiers supérieurs et une plus grande vulnérabilité aux variations de la politique monétaire. 
Logiquement, ce panorama déficient ne concerne pas uniquement le cas espagnol ; mais du point de vue analytique, il peut exister des raisons particulières qui l'expliquent étant donné que ce qui nous intéresse est de trouver les causes de cette situation en Espagne. En d'autres termes, il faut trouver les raisons de l'existence de tels problèmes, car ils influent sur la compétitivité de l'économie espagnole. Si l'on écarte les caractéristiques propres à l'entrepreneur, lesquelles seront traitées dans la section suivante, une des principales causes se trouve dans la nature particulière de la structure entrepreneuriale espagnole.

Tout d'abord, le secteur secondaire présente une forte atomisation, dont les principales caractéristiques sont les suivantes : une taille moyenne des entreprises de 12,8 employés (tableau 3) et un nombre élevé d'entreprises à caractère familial, lesquelles représentent plus de la moitié de l'effectif total ; proportion très supérieure, selon certaines études empiriques, à celle des économies voisines (France, Italie), où le poids de ce type d'entreprises n'atteint pas le tiers du total du secteur (Fariñas et al., 1992). En conséquence, la plupart des entreprises industrielles se retrouvent dépendantes de l'évolution des entreprises publiques ou des grands groupes leaders, exerçant un rôle d'industrie connexe par rapport à ceux-ci. De plus, la production de certains soussecteurs, comme ceux de l'industrie agro-alimentaire et du textile, doit s'adapter aux orientations données par les hypermarchés et les centres commerciaux. Mais la principale faiblesse du tissu industriel espagnol s'explique par l'excès de spécialisation des entreprises dans les secteurs à faible demande. En effet, d'après le tableau 4, plus de la moitié des entreprises industrielles se concentrent dans les sous-secteurs de production traditionnelle, où la demande est faible (métallurgie, textile, etc.). Alors que les sous-secteurs à forte demande (informatique, électronique, etc.), qui proportionnellement ont plus d'employés, ne représentent pas plus du dixième de l'ensemble des entreprises industrielles du pays.

Ensuite, comme cela se passe dans la majorité des pays relativement avancés, l'atomisation des entreprises se manifeste davantage dans le secteur tertiaire. Comme on peut le constater dans le tableau 3, les entreprises de services atteignent $70 \%$ du total et leur taille moyenne (hors services publics) est de 6 employés. Mais dans notre cas, cette forte atomisation présente quelques conséquences transcendantes : bien que d'un point de vue macro-économique l'importance apparente du secteur des services soit assez élevée (plus de $60 \%$ de l'emploi dans le pays), la petite taille des entreprises fait que des soussecteurs traditionnels de faible valeur ajoutée prédominent, en particulier ceux dont les caractéristiques fonctionnelles sont fortement liées à la demande finale et, par conséquent, en très faible relation avec le reste de l'appareil productif. 
TABleau 3

Distribution entrepreneuriale par secteurs

\begin{tabular}{lccc}
\hline Secteurs & $\begin{array}{c}\text { Pourcentage } \\
\text { d'entreprises }\end{array}$ & $\begin{array}{c}\text { Pourcentage } \\
\text { d'emplois }\end{array}$ & $\begin{array}{c}\text { Taille moyenne par } \\
\text { entreprises } \\
\text { (nombre de salariés) }\end{array}$ \\
\hline Agriculture et pêche & 0,5 & 0,3 & 7,4 \\
Industrie & 18,4 & 27,2 & 12,8 \\
Construction & 11,8 & 11,7 & 13,5 \\
Services $^{1}$ & 69,7 & 60,7 & 6,0 \\
\hline
\end{tabular}

1. À l'exclusion des services publics.

Source : Sécurité sociale.

Tableau 4

Structure sectorielle de l'industrie, selon le type de demande

Classification des sous-secteurs

$\begin{array}{cc}\begin{array}{c}\text { Nombre } \\ \text { d'entreprises }\end{array} & \begin{array}{c}\text { Pourcentage } \\ \text { d'emplois } \\ \text { Total national }\end{array} \\ \begin{array}{c}\text { Total national } \\ (\%)\end{array} & (\%)\end{array}$

Demande forte

Équipement de bureau

Produits électriques et électroniques

Produits chimiques et pharmaceutiques

$\begin{array}{ll}0,02 & 0,1 \\ 1,1 & 2,1 \\ 1,2 & 2,2 \\ 2,3 & 4,4\end{array}$

Sous-total

2,3

4,4

Demande moyenne

Produits de plastique

$0,3 \quad 0,8$

Équipement de transport

$0,2 \quad 2,3$

Alimentation, boissons et tabac

$2,6 \quad 3,8$

Papier

1,3

1,9

Équipement agricole et industriel

0,6

1,0

Sous-total

5,3

9,8

Demande faible

Produits métallurgiques

$3,9 \quad 4,5$

Produits manufacturés variés

Textiles

3,1

2,8

2,4

Minéraux non métallurgiques

1,0

4,1

Sous-total

10,8

12,9

Total pour le secteur industriel

18,4

27,2

1. Selon la Commission européenne.

Source: Sécurité sociale. 
TABleau 5

Principaux sous-secteurs de services en Espagne

\begin{tabular}{lcccc}
\hline Sous-secteur & $\begin{array}{c}\text { Pourcentage } \\
\text { d'entreprises } \\
\text { Total } \\
\text { national }\end{array}$ & $\begin{array}{c}\text { Pourcentage } \\
\text { d'emplois } \\
\text { Total } \\
\text { national }\end{array}$ & $\begin{array}{c}\text { Pourcentage } \\
\text { de petites } \\
\text { entreprises }\end{array}$ & $\begin{array}{c}\text { Type } \\
\text { prédominant }\end{array}$ \\
\hline Commerce de détail & 18,1 & 9,2 & 99,2 & $\mathrm{c}-\mathrm{b}$ \\
Restaurants et cafés & 8,1 & 3,3 & 99,5 & $\mathrm{c}$ \\
Commerce en gros & 7,0 & 5,1 & 98,7 & $\mathrm{a}$ \\
Services aux entreprises & 5,2 & 4,1 & 98,1 & $\mathrm{a}$ \\
Transports terrestres & 4,3 & 2,6 & 98,8 & $\mathrm{a}-\mathrm{c}$ \\
Services personnels & 3,6 & 1,3 & 99,2 & $\mathrm{c}$ \\
Réparation & 3,0 & 1,7 & 99,1 & $\mathrm{c}$ \\
\multicolumn{1}{c}{ Sous-total } & 49,3 & 27,3 & 99,2 & \\
Reste services (publics) & 9,9 & 22,4 & & \\
Reste services (privés) & 10,5 & 11,0 & & \\
\hline
\end{tabular}

1. Moins de 50 salariés.

2. $\mathrm{a}=$ satellites $; \mathrm{b}=$ opposition dominée $; \mathrm{c}=$ indépendantes.

Source : Sécurité sociale.

Comme le montre le tableau 5, il y a sept activités traditionnelles de services qui englobent quasiment la moitié des entreprises du pays $(49,3 \%)$, mais qui ne représentent que le quart du total des emplois. L'importance quantitative du secteur des services dans l'ensemble de l'emploi national reste cependant renforcée par les services publics $(22,4 \%)$ sans lesquels le poids de "l'initiative privée » du secteur tertiaire en Espagne serait considérable par son nombre d'entreprises, mais pas pour son apport en PIB et en emploi.

On peut trouver une troisième cause de la faiblesse du tissu productif espagnol dans la typologie fonctionnelle des petites entreprises. Pour cela, en ce qui nous concerne, nous pouvons trouver une optique opérationnelle dans la classification des PME que Averitt a exposée il y a déjà plus de vingt-cinq ans. Évidemment, l'exposé de Averitt n'est pas le seul, ni le plus précis, mais son optique analytique, pour le cas espagnol du moins, peut encore posséder suffisamment de vigueur pour expliquer la situation actuelle. Selon Averitt (1968), la grande entreprise tient un rôle d' « économie centrale », alors que la PME agit en tant qu' « économie entrepreneuriale périphérique ». À partir de ce concept, on distingue trois grandes catégories de PME, bien différenciées.

1. Les PME «satellites». Elles possèdent des liens de dépendance envers une grande entreprise, dont dépendront leur fonctionnement et leur existence.

2. Les PME «d'opposition dominée ». Elles sont géographiquement localisées sur le même marché que la grande entreprise. Des choix de 
cette dernière dépendront le comportement de la PME ainsi que sa politique de prix et son aptitude à créer de nouveaux produits.

3. Les PME «indépendantes». Que ce soit contractuellement ou tacitement, elles ne maintiennent aucun lien avec la grande entreprise. Ces PME agissent sur des marchés réduits et très différenciés.

En termes quantitatifs, la première catégorie de PME fonctionne grâce à la sous-traitance et, de plus, assure la distribution et la vente des produits fabriqués par les grandes entreprises. L'activité de ce type de PME se situe en général dans les secteurs connexes de l'industrie, mais aussi dans la vente en gros et dans certaines branches des secteurs de la construction et des transports. En ce qui concerne les PME « d'opposition dominée », elles se spécialisent dans le commerce de détail, et sont installées dans les zones urbaines proches des grandes surfaces et des centres commerciaux. Enfin, les PME « indépendantes » regroupent les activités du petit commerce de détail, de l'hôtellerie, de la prestation de services aux particuliers, des petits ateliers de réparation, de la construction individuelle et de l'industrie artisanale.

À partir de cette typologie, nous avons pu réaliser que la quatrième colonne du tableau 5 représente une application approximative de la théorie d'Averitt à la réalité entrepreneuriale du secteur le plus important de l'économie espagnole. Et même si les données disponibles ne permettent pas de classer toutes les entreprises dans l'une des trois catégories, il apparaît que les $\mathrm{PME}$ « indépendantes » de services représentent au moins un tiers de l'ensemble des entreprises espagnoles. En y ajoutant les petites entreprises artisanales et celles de la construction individuelle, logiquement éloignées dans la majorité des grandes concentrations urbaines, il paraît clair que les PME « indépendantes » représentent au moins la moitié de la population entrepreneuriale en Espagne. De même, et en considérant plus particulièrement les activités de sous-traitance et de l'industrie connexe - normalement caractérisées par des activités traditionnelles très étendues dans les secteurs industriels pour lesquels la demande est moyenne et faible -, les PME « satellites » regroupent au moins un cinquième des entreprises du pays (tableaux 3, 4 et 5). Tout cela, sans considérer le poids du travail au noir qui, selon certaines estimations, représente $15 \%$ du PIB. Dans la plupart des cas, il s'agit d'activités de soustraitance, de petits commerces, d'ateliers de réparation, etc.

À première vue, le nombre élevé de petites entreprises « indépendantes » peut constituer un des aspects positifs de l'appareil productif espagnol, face à la vulnérabilité qu'entraîne une excessive dépendance des PME envers les grands groupes. Cependant, cet avantage perd de sa valeur si l'on se réfère à l'approche d'Averitt et à son application à la réalité espagnole. Selon ses critères, ces petites entreprises «indépendantes » se spécialisent généralement 
dans des secteurs à faible demande. Et même si cette demande est fidèle et inélastique, en raison de la nature de ses outputs (biens et services de base) ou de l'absence de grandes entreprises sur ces marchés, ou encore en raison de sa faible sensibilité à la conjoncture économique, elle reste insuffisante. En d'autres termes, il s'agit de petites unités productives, fortement isolées, et peu exposées à la concurrence nationale et internationale. De ce fait, malgré une faible intégration des technologies modernes et la présence de coûts financiers et salariaux élevés, elles réussissent à se maintenir en vie.

\section{Point de vue qualitatif de l'entrepreneur espagnol}

Dans l'une des rares études empiriques établissant une typologie de l'entrepreneur espagnol apparaissent les différentes catégories suivantes (Velarde, 1993) : 1) les entrepreneurs libéraux, ayant bien accepté les règles du libreéchange ; 2) les entrepreneurs protégés, profitant de l'appui des actions protectionnistes de l'État ; 3) les entrepreneurs financiers ; 4) les entrepreneurs étrangers.

D'un point de vue historique, les principales faiblesses de l'appareil productif espagnol remontent au XIX ${ }^{\mathrm{e}}$ siècle. Elles s'expliquent, d'abord, par le manque d'entrepreneurs libéraux participant à l'activité économique. En outre, le système juridique institutionnel a favorisé, durant des décennies, l'existence d'un marché excessivement protégé et, par conséquent, incapable de développer un processus d'internationalisation de l'entreprise espagnole (Arruñada, 1993).

Mais ce type d'étude traite plus particulièrement des relations entre les entrepreneurs et les pouvoirs publics, c'est-à-dire de l'environnement extérieur de l'entreprise et non pas des caractéristiques endogènes de celle-ci. Il est évident que les facteurs exogènes, politiques ou économiques, influent sur les décisions prises par l'entrepreneur. Par contre, le comportement et l'efficacité des entreprises de petite taille sont en grande partie déterminés par les caractéristiques de «l'entrepreneur ». Nous avions déjà mentionné le manque d'intérêt porté à la figure de l'entrepreneur. Le retard de la recherche empirique dans ce domaine est tel qu'il n'existe toujours pas de configuration d'une méthodologie comparative appropriée.

Logiquement, une étude approfondie des traits caractéristiques de l'entrepreneur ne peut se faire sans utiliser un concept théorique le définissant (Herbert et Link, 1989). Néanmoins, sans nous attarder à une analyse conceptuelle de l'entrepreneur, nous pourrons nous référer aux composantes essentielles de sa fonction dans le but de réaliser une approche de la «qualité » 
de l'entrepreneur espagnol. Ainsi nous pouvons diviser ces composantes en trois sphères fondamentales (Guzman, 1994) :

1) la sphère «capitaliste », assimilable à la propriété des ressources propres ;

2) la sphère «gestion », où se développe l'organisation et s'élabore la prise de décision;

3) la sphère « impulsive », qui regroupe les caractéristiques psychologiques et sociologiques de l'entrepreneur, permettant la création et le maintien en vie de l'unité productive. C'est ce qu'on appelle communément l' « esprit d'entreprise ».

À partir des informations dont nous disposons sur les fonctions « gestion » et « impulsive », nous tenterons d'approfondir la connaissance de la qualité d'entrepreneur.

En ce qui concerne la fonction «gestion », la majorité des entreprises espagnoles présentent des déficiences organisationnelles ainsi qu'une capacité technologique et commerciale insuffisante (Martin, 1993). Cependant, dans la catégorie des micro-entreprises et des petites entreprises de moins de 50 salariés, les fonctions «gestion » sont principalement assumées par l'entrepreneur, si bien que la qualité de la gestion pratiquée sera en grande partie déterminée par la formation de celui-ci. Selon le tableau 6, la formation des entrepreneurs espagnols peut être qualifiée de déficiente, plus de la moitié n'ayant pas terminé leur primaire. Mais la situation semble s'améliorer assez rapidement, puisqu'en moins de dix ans la proportion des entrepreneurs dépassant ce niveau d'études est passée de 36,2\% à 47,3\%. De plus, ce processus d'amélioration technique de la gestion des entreprises est irréversible. Cela s'explique par l'augmentation du nombre d'agents de direction et de spécialistes offrant leurs services sur le marché du travail.

TABleau 6

Évolution de la formation de l'entrepreneur espagnol ( \%)

\begin{tabular}{lrr}
\hline Niveau d'études & $\mathbf{1 9 8 1}$ & $\mathbf{1 9 8 9}$ \\
\hline Analphabètes & 0,5 & 0,2 \\
Sans études & 4,2 & 6,1 \\
Niveau primaire & 59,2 & 46,4 \\
Niveau secondaire & 23,0 & 31,5 \\
Niveau intermédiaire & 3,9 & 5,8 \\
Niveau universitaire & 9,3 & 10,0 \\
Total & 100,0 & 100,0 \\
\hline
\end{tabular}

Source: Enquête sur la population active. 
L'étude de la sphère «impulsive », propre à l'entrepreneur espagnol, nous oblige à résoudre deux types de problèmes. Tout d'abord, il s'agit des difficultés habituellement rencontrées lors de la recherche de données et de sources d'information. Puis, dans un second temps, s'ajouteront d'autres problèmes découlant de la grande hétérogénéité des économies régionales. En effet, il existe une faible relation entre les centres de forte tradition entrepreneuriale (Catalogne, Pays basque) et les zones plus faiblement industrialisées.

Ainsi, une étude relative à la nature de l'entrepreneur basque (Fernández, 1990) met en valeur certaines caractéristiques positives, telles que l'esprit d'initiative, la créativité, le goût du risque, la responsabilité professionnelle, etc.

Mais en marge de cette zone géographique de forte tradition entrepreneuriale qui, avec Madrid et Barcelone, regroupe la majorité des grandes entreprises espagnoles (voir le tableau 2) subsistent plusieurs régions où l'esprit d'entreprise n'est pas valorisé. Et l'on affirme même que dans le reste de l'Espagne, «il y a peu d'entrepreneurs».

En effet, le triangle Madrid-Barcelone-Pays basque absorbe à lui seul plus des deux tiers des grandes entreprises espagnoles. Quant au reste du pays, il s'apparente à un « désert » d'initiatives entrepreneuriales. Cependant, l'analyse du volume de la population entrepreneuriale réelle par régions (tableau 7) révèle que ce problème se situe plutôt sur le plan qualitatif : Madrid, la Catalogne et le Pays basque regroupent les plus grandes entreprises (tableau 2), mais elles ne possèdent pas un nombre supérieur d'entrepreneurs.

Cet aspect qualitatif des faiblesses entrepreneuriales apparaît aussi dans d'autres travaux de recherches empiriques menées en Andalousie sur l'entrepreneur du Sud de l'Espagne. En schématisant, on peut distinguer les points faibles suivants (Vallés et al., 1988 ; Guzmán, 1990) :

1. le « faible niveau d'association et d'esprit de collaboration »;

2. le manque d'ambition et de capacité de croissance ;

3. la faible motivation à la réussite ;

4. l'insuffisante préoccupation concernant l'autofinancement.

Ces points faibles se rencontrent aussi dans les segments des petites entreprises, dans la majeure partie du secteur productif espagnol, et plus particulièrement dans les activités de services, où la création d'entreprise est plus facilement réalisable et technologiquement plus simple que dans le secteur industriel (Arias et al., 1994). Si l'on tient compte des caractéristiques du tissu productif espagnol déjà mentionnées (atomisation, prédominance des services, entreprises « indépendantes », etc.), il semble clair que la majorité de la popu- 
lation entrepreneuriale s'apparente plus à l'entrepreneur du Sud de l'Espagne qu'à celui des zones plus industrialisées. De ce fait, les rares recherches empiriques réalisées dans l'ensemble du pays démontrent l'existence d'un faible " esprit d'entreprise », visant particulièrement les motivations à la création d'entreprises (Lafuente et Salas, 1989). Ce problème peut avoir des conséquences importantes dans le contexte européen. Selon certaines études, les entrepreneurs français et italiens semblent adopter une attitude positive face au défi imposé par le marché européen. Ce n'est pas le cas de l'Espagne qui, en plus de son manque de prise de conscience, se désintéresse des organismes et des services mis à la disposition des PME afin d'accéder à l'information dont elles ont besoin (Ernst et Young, 1990).

\section{TABLEAU 7}

Population entrepreneuriale par régions (1991)

\begin{tabular}{lcc}
\hline Régions & $\begin{array}{c}\text { Distribution par } \\
\text { pourcentage d'entrepreneurs }\end{array}$ & $\begin{array}{c}\text { Pourcentage d'entrepreneurs } \\
\text { sur population occupée }\end{array}$ \\
\hline Andalousie & 14,5 & 20,5 \\
Aragon & 4,1 & 25,5 \\
Asturies & 3,1 & 22,5 \\
Baléares & 2,2 & 23,0 \\
Canaries & 2,7 & 14,9 \\
La côte cantabrique & 1,4 & 21,9 \\
Castille-et-Léon & 9,4 & 28,8 \\
Castille-la-Manche & 5,3 & 25,0 \\
Catalogne & 14,7 & 17,0 \\
Valencia & 10,3 & 20,6 \\
Extremadure & 3,0 & 25,6 \\
Galice & 12,7 & 32,4 \\
Madrid & 7,1 & 11,1 \\
Murcie & 2,2 & 17,6 \\
Navarre & 1,6 & 22,0 \\
Pays basque & 4,7 & 16,9 \\
La Rioja & 0,9 & 26,5 \\
Espagne & 100,0 & 21,5 \\
\hline
\end{tabular}

Source : Enquête sur la population active, Institut national de statistiques.

En définitive, l'étude des caractéristiques générales propres à l'entrepreneur et l'analyse du type d'entreprises dominant en Espagne nous permettent d'expliquer les raisons poussant à la création de petites unités, telles que les micro-entreprises et les petites entreprises. En effet, leur présence s'explique plus par la nécessité de survivre que par les critères avancés par les différentes théories (Schumpeter, Kirzner, Knight, etc.) traitant de l'émergence de l'activité entrepreneuriale. 


\section{3. Évaluation des politiques de soutien aux PME}

En Espagne, comme dans la plupart des pays de son environnement économique, il existe une large gamme de mesures de soutien aux petites et moyennes entreprises. On en relève plus d'une centaine, si l'on compte celles de la Communauté européenne, de l'administration centrale, des communautés autonomes et des administrations locales (députations provinciales et mairies).

Malgré l'existence de ce large éventail de mesures et d'institutions visant le soutien aux PME, dans la pratique les effets de ces mesures sont assez faibles d'un point de vue de la régénérescence du tissu entrepreneurial et, par conséquent, de l'appareil productif du pays. De façon schématique, les raisons de ce manque d'efficience sont les suivantes :

1. La majorité des mesures de soutien sont de caractère financier (crédits préférentiels, subventions, déductions fiscales, etc.) et se basent exclusivement sur l'entreprise, sans considérer le point de vue de l'entrepreneur. Cela suppose donc que l'on aborde le problème de la structure entrepreneuriale depuis la base, c'est-à-dire les facteurs ayant une incidence sur la culture qualitative des entrepreneurs.

2. L'insuffisance de coordination entre les différentes institutions destinées à appuyer les PME. Devant les fréquents « chevauchements » des directives, il serait nécessaire d'appliquer des politiques d'aide technologique, de financement, de formation et de stimulation à la culture entrepreneuriale, établies depuis l'administration centrale.

3. Selon les entrepreneurs, il existe trop d'obstacles bureaucratiques au développement entrepreneurial, surtout dans les premières phases de création et de mise en marche de PME (Fontela, 1994).

4. La majeure partie de ces programmes de soutien a été pensée pour des entreprises de 50, 100 ou 200 employés, déterminant des objectifs et prévoyant, par exemple, la création de 10 postes de travail, d'importants changements technologiques ou des quotas d'exportation élevés. Il est donc évident qu'il y a un manque de réalisme dans ce type de programmes d'aide aux PME.

En effet, ces politiques d'aide aux PME devraient dépasser le stade de ce que nous avons appelé les sphères «capitalistes » et « gestion » de la fonction entrepreneuriale. Les aides financières ou fiscales, même les cours et les «masters » de formation, ne permettent pas de développer la sphère « impulsive » des entrepreneurs actuels et potentiels. En fait, la transformation de la structure entrepreneuriale ne pourra se faire sans une politique de soutien aux petites et aux moyennes entreprises, incluant une vision inspirée des principes 
holistiques, c'est-à-dire en considérant le système économique comme un ensemble de normes de comportement de caractère institutionnel et culturel, dans lequel il existe une sphère d'influence mutuelle entre les aspects économiques et sociaux.

\section{Bibliographie}

ARIAS, C. et al. (1994), « Una aproximación a la calidad funcional del empresario », VIII Congreso Asepelt, Palma de Mallorca.

ARRUÑADA SÁNCHEZ, B. (1993), «Una explicación contractual del fracaso de la empresa española », Documento de trabajo, Fundación FIES, n 94 , Madrid.

Averitt, R. (1968), The Dual Economy, New York, Norton and Company.

BANCO DE EsPAÑA (1990), Central de Balance.

ERnst et Young (1990), Las Pymes y el Mercado Unico. El Reto de su Competitividad, Madrid, IMPI .

FARIÑAS, J.C. et al. (1992), La Pyme Industrial en España, Madrid, IMPI, Cívitas.

FERnÁndez Monge, F. (1990), « Estudio cualitativo del empresario vasco », Círculo de Empresarios, Boletín 51, p. 95-117.

FONTELA, E. (1994), « La relación entre las pequeñas empresas y las administraciones públicas », Congreso de la Pequeña Empresa, San Sebastián, ADEGI, p. 2023.

Guzmán Cuevas, J. (1986), El Sistema Financiero y las Pymes Andaluzas, Sevilla, Instituto de Desarrollo Regional.

GuzMÁn Cuevas, J. et M. PAZ BÁÑEz (1991), « The entrepreneurial promotion in depressed areas : the case of Southern Spain », 31 RSA European Congress, Lisbon.

GuZMÁn CUEVAS, J. (1994), "Towards a taxonomy of entrepreneurial theories », International Small Business Journal, vol. 12, $\mathrm{n}^{\circ} 4$, p. 77-88.

HERBERT, R.F. et A.N. LINK (1989), «In search of the meaning of entrepreneurship », Small Business Economics, vol. 1, p. 39-49.

JORDÁ BORREL, R.M. (1992), Las Relaciones Ciencia-Tecnología-Industria en Andalucía y el papel de la Administración, Sevilla, I.D.R.

JULIEN, P.A. (1989), «The entrepreneur and economic theory », International Small Business Journal, vol. 7, $\mathrm{n}^{\circ}$ 3, p. 29-38.

LAFUENTE, A. et V. SAlas (1989), « Types of entrepreneurs and firms : the case of new Spanish firms », Strategic Management Journal, vol. 10, p. 17-30. 
MARTín, C. (1993), «La empresa y la competitividad de la economía española », Cuadernos de Información Económica, Madrid, fundación FIES, p. 11-15.

SMITH, N.R. et J.B. MINER (1983), « Type of entrepreneur, type of firm, and managerial motivation: implications for organizational life cycle theory », Strategic Management Journal, vol. 4, p. 325-340.

VAllés FerRer, J. et al. (1988), « Hacia un perfil cualitativo del empresario en Andalucía », Junta de Andalucía.

Veciana, J.M. (1989), "Características del empresario en España », Papeles de Economía, nos 30-40, Madrid, p. 19-36.

VElarde FUERTES, J. (1993), «Talante y realidades empresariales en la industrialización española », dans Empresas y Empresarios Españoles en la Encrucijada de los Noventa, Madrid, Cívitas. 